Article

\title{
Structure-Based Virtual Screening of Novel Natural Alkaloid Derivatives as Potential Binders of $h$-telo and $c$-myc DNA G-Quadruplex Conformations
}

\author{
Roberta Rocca ${ }^{1, \dagger}$, Federica Moraca ${ }^{1, \dagger}$, Giosuè Costa ${ }^{1}$, Stefano Alcaro ${ }^{1, *}$, Simona Distinto ${ }^{2}$, \\ Elias Maccioni ${ }^{2}$, Francesco Ortuso ${ }^{1}$, Anna Artese ${ }^{1}$ and Lucia Parrotta ${ }^{1}$
}

1 Dipartimento di Scienze della Salute, Università degli Studi "Magna Græcia", Campus "S. Venuta", Viale Europa, Germaneto 88100, Catanzaro, Italy; E-Mails: rocca@unicz.it (R.R.); fmoraca@unicz.it (F.M.); gcosta@unicz.it (G.C.); ortuso@unicz.it (F.O.); artese@unicz.it (A.A.); 1parrotta@unicz.it (L.P.)

2 Dipartimento di Scienze della Vita e dell'Ambiente, Università degli Studi di Cagliari, Via Ospedale 72, Cagliari 09124, Italy; E-Mails: s.distinto@unica.it (S.D.); maccione@unica.it (E.M.)

$\dagger$ These authors contributed equally to this work.

* Author to whom correspondence should be addressed; E-Mail: alcaro@unicz.it; Tel.: +39-0961-369-4197; Fax: +39-0961-369-4073.

Academic Editors: Patricia Valentao and Mariana Sottomayor

Received: 7 November 2014 / Accepted: 15 December 2014 / Published: 24 December 2014

\begin{abstract}
Several ligands can bind to the non-canonical G-quadruplex DNA structures thereby stabilizing them. These molecules can act as effective anticancer agents by stabilizing the telomeric regions of DNA or by regulating oncogene expression. In order to better interact with the quartets of G-quadruplex structures, G-binders are generally characterized by a large aromatic core involved in $\pi-\pi$ stacking. Some natural flexible cyclic molecules from Traditional Chinese Medicine have shown high binding affinity with G-quadruplex, such as berbamine and many other alkaloids. Using the structural information available on G-quadruplex structures, we performed a high throughput in silico screening of commercially available alkaloid derivative databases by means of a structure-based approach based on docking and molecular dynamics simulations against the human telomeric sequence $d\left[\mathrm{AG}_{3}\left(\mathrm{~T}_{2} \mathrm{AG}_{3}\right)_{3}\right]$ and the $c$-myc promoter structure. We identified 69 best hits reporting an improved theoretical binding affinity with respect to the active set. Among them,
\end{abstract}


a berberine derivative, already known to remarkably inhibit telomerase activity, was related to a better theoretical affinity versus c-myc.

Keywords: DNA; G-quadruplex; h-telo; c-myc; alkaloids; berberine; virtual screening; docking; molecular dynamics

\section{Introduction}

Non-canonical high-order DNA structures, known as G-quadruplexes (G4s), are formed by guanine-rich DNA sequences and are prevalent in the human genome. Bioinformatics analyses show that $\mathrm{G} 4 \mathrm{~s}$ sequence motifs are present in many important regions of the eukaryotic genome, such as telomere ends and the regulatory regions of several oncogenes, including c-myc, c-kit, V-Ki-ras2 Kirsten rat sarcoma viral oncogene homolog (KRas), platelet-derived growth factor subunit A (PDGF-A), vascular endothelial growth factor (VEGF), intron regions and the insulin-related polymorphism regions [1-11]. Recently G4s, that demonstrated an important role in vivo [12-14], were considered as potential targets for cancer therapy [15-17].

The presence of monovalent cations, such as $\mathrm{Na}^{+}$and $\mathrm{K}^{+}$, and a network of Hoogsteen hydrogen bonds are responsible for the stabilization of the stacked G-tetrad planes that form the G4 structures. Polymorphism is an essential feature of the G4 DNA that assumes various conformations, depending on the counterion type, incubation period and sequence [18]. In particular, the G4s strands intertwine to form parallel, anti-parallel or mixed hybrid conformations which result in a variety of different grooves and loops [19-22]. The presence of dimeric G4 structures, besides the formation of monomeric intermolecular and intramolecular conformations, is also reported. Specifically, the telomeric G4 DNA can either occur by dimerization of hairpin loops or by directly folding into intramolecular structures [23,24]. Regarding the importance of G4 structures, specific ligands that can induce their formation and stabilization are considered as potential drugs due to their inhibitory action on telomerase activity and oncogene expression $[25,26]$. The telomerase enzyme, normally absent in somatic cells but present in $85 \%-90 \%$ of cancer cells, is able to add the telomeric repeat unit (TTAGGG in case of humans) to the ends of the chromosome contributing to their immortality [27-29]. In order to achieve such an aim, the telomeric region of the chromosome must remain single stranded [30]. Thus, by either blocking telomerase directly or by making the telomerase substrate inaccessible, the uncontrolled cellular proliferation can be stopped. The most interesting therapeutics are small molecule inhibitors, antisense oligonucleotides, immunotherapies, gene therapies, telomere and telomerase associated protein inhibitors, T-oligo and G4 stabilizers. In particular, although there are various selective synthetic and natural G4 molecules, with different chemical scaffolds, widely studied also by our research group [31-38], three of the most commonly investigated G4 stabilizing agents are the trisubstituted acridine BRACO-19, the polycyclic compound RHPS4 and the natural ligand telomestatin $[39,40]$.

In the human genome there are $\sim 376,000$ potential G4 forming motifs, as shown by bioinformatics studies $[9,11]$. These motifs, abundant in the promoter region of genes especially near the transcription start site (c-myc, c-kit, c-myb, KRAS, PDGF-A, VEGF, BCL-2, MyoD, HIF-1 $\alpha$ and TK1) suggested a key role of G4 structures in transcription [41]. Among these, the quadruplex in the promoter region 
of $c$-myc is widely investigated. A variety of malignant tumors are associated with the aberrant over-expression of c-myc [42]. This region contains a cytosine-rich (C-rich) coding strand and a guanine-rich (G-rich) non-coding strand that are capable of engaging in a slow equilibrium between B-form duplex DNA, single-stranded DNA, and tetra-stranded DNA. Specifically, the cytosine tracts in the complementary $\mathrm{C}$-rich strand can form an i-motif or i-tetraplex, whereas the guanine tracts of the G-rich strand can form a G4 structure [43]. The nuclear hypersensitivity element $\mathrm{III}_{1}\left(\mathrm{NHE} \mathrm{III}_{1}\right)$, upstream of the P1 promoter of $c-m y c$, controls $80 \%-90 \%$ of the $c-m y c$ transcription level $[44,45]$. The NHE III $_{1}$, a G-rich strand of the DNA containing 27 base-pair sequence, is able to form intramolecular G4 structure and functions as a transcriptional repressor element $[43,46,47]$. The first intramolecular G4 structure within the $c$-myc NHE III 1 region was proposed by Simonsson et al. [45] that described an antiparallel-stranded structure involving three G-tetrads formed from four G-tracts linked by two lateral loops and a central diagonal loop. However, in the $c$-myc NHE III 1 region further studies, carried out by chemical footprinting, circular dichroic (CD) and nuclear magnetic resonance, revealed that there is a single G4 isomer of parallel topology containing three lateral loops (a 1:2:1 loop isomer) [48]. The G4 present in the NHE III 1 region of the $c$-myc promoter was shown to function as a silencer element [46]. Consequently, the approach to design specific inhibitors able to stabilize this structure could potentially be an effective approach of targeting human malignancies that overexpress $c$-myc. In fact, Grand and collaborators showed that the cationic porphyrinTMPyP4 decreases $c-m y c$ expression at both the mRNA and protein levels and reduces the level of several c-myc-regulated genes [49]. Moreover, different natural products derived from Traditional Chinese Medicine have been found associated to high binding affinities versus diverse G4 sequences, such as berbamine, which is a natural alkaloid able to better stabilize (GGA) ${ }_{8}$ G4 compared with other six G4s [50].

Several studies related to the rational drug design of natural molecules have been performed by means of structure-based techniques. In particular, this approach, complementing conventional high-throughput techniques, represents an efficient strategy in drug discovery [51-57]. The number of compounds synthesized and tested in vitro can be dramatically reduced through the identification of inactive non-binders in silico. Meanwhile, an important source of bioactive substructures and chemical scaffolds can be found in natural products [58].

Recently, Gosh et al. applied absorption and fluorescence studies, demonstrating that sanguinarine, a naturally occurring plant alkaloid isolated from Sanguinaria canadensis [59], had high binding affinity towards two quadruplex forming sequences, human telomeric DNA (H24) and NHE III1 upstream of the $\mathrm{P} 1$ promoter of $c-m y c$ ( $\mathrm{Pu} 27$ ). Specifically, by means of differential scanning calorimetry studies, sanguinarine binding was found to increase the melting point and also the total enthalpy of transition of both quadruplexes, while TRAP results showed that it effectively blocked telomerase activity in a concentration dependent manner [60].

Sanguinarine and other 10 alkaloids were investigated by Ji et al. for their ability to interact with G4 formed by telomeric DNA and $C$-myc22 sequences through molecular modeling and biophysics experiments. In particular, sanguinarine, palmatine and berberine were observed to induce the formation of G4 as well as to stabilize it due to the presence of unsaturated ring, positively charged nitrogen centers, and conjugated aromatic rings [61].

Berberine, an alkaloid isolated from Chinese herbs, was initially used as anti-microbial agent; nowadays it represents a hot research topic, since it demonstrates various pharmacological activities that have 
applications in several therapeutic areas, such as cancer, diabetes, depression, cardiovascular, and hypertension [62-64]. In 2012, Bessi and co-workers, by means of fluorescence, CD, molecular modeling and NMR studies, demonstrated the interactions of the natural alkaloids berberine and sanguinarine with basket- and hybrid-type h-telo G4 DNA with a high stoichiometry as a consequence of specific binding modes [65].

Recently, 13-substituted berberine derivatives were reported by Neidle's group for their abilities to selectively bind to G4 over double-stranded DNA, and inhibited telomerase activity by binding to G4 DNA [66,67]. Later, Zhang et al. demonstrated that 9-O-substituted berberine derivatives could induce and stabilize the anti-parallel G4 structure in the telomere DNA independently of the presence of metal cations [68]. Subsequently, Ma et al. designed and synthesized a series of 9-N-substituted berberine derivatives and investigated their interactions with $\mathrm{NHE} \mathrm{III}_{1}$ DNA by means of electrophoretic mobility shift assay (EMSA), circular dichroism spectroscopy (CD), fluorescence resonance energy transfer-melting (FRET-melting) method, polymerase chain reaction-stop assay (PCR-stop assay), competition dialysis method, cell proliferation assay and reverse transcription-polymerase chain reaction (RT-PCR). All the results showed that the 9-N-substituted berberine derivatives could down-regulate the transcription of $c$-myc in cancer cell line by selectively stabilizing the formation of intramolecular parallel G4 in c-myc DNA [69].

Then, the same research group, by adding a pyridine ring and an amino group into berberine, improved the binding ability and selectivity towards G4 DNA in comparison with the previously reported 9-N-substituted berberine derivatives [70]. The synthesized quinoline benzo[5,6]dihydroisoquindolium compounds also showed good inhibition against $c-m y c$ transcription in tumor cells only.

More recently, Bournine et al. reported the anti-tumoral activity of Glaucium flavum root alkaloid extract against human cancer cells, in vitro and in vivo, highlighting protopine as the principal alkaloid identified by HPLC-DAD. Finally, the authors demonstrated a specific anticancer effect of Glaucium flavum root extract against breast cancer cells, which can be, at least in part, attributed to the other identified alkaloid bocconoline [71]. Recent ESI-MS analyses, performed by Cui et al. [72], reported three natural flexible cyclic molecules, tetrandrine, fangchinoline and cepharanthine, which can bind to the intermolecular and intramolecular RNA G4s from $r$ (UAGGGUUAGGGU) and $r$ (UAGGGUUAGGGUUAGGGUUAGGGU) sequences with high affinity.

All these studies highlight that the literature on alkaloids is growing rapidly. Researchers are persistently attempting to decode the many secrets surrounding alkaloids, since they believe that drugs developed from alkaloids or by using natural models of these compounds could help in the search for future cures to serious diseases, such as cancer or AIDS [73].

With respect to anticancer activity, we reported above many biophysical and biochemical studies which have shown that the natural benzophenantridine alkaloid berberine and its derivatives bind to human telomeric G4 structures, the $c$-myc oncogene and other biologically relevant G-rich sequences $[67,69,74]$. The interest toward this scaffold and G4 target lead Bazzicalupi et al. to solve the first crystal structure of human telomeric DNA in complex with the natural alkaloid berberine by $\mathrm{X}$-ray diffraction method [75]. It was observed the presence of two berberine molecules in the two binding sites, directly interacting with each tetrad. 
It is widely known that most of the G4-interactive compounds are planar molecules with multiple condensed rings that intercalate between the G-tetrad and the flanking residues of the pre-formed "drug binding pockets" [3,76-79].

Although there is a growing list of molecular structures reported for G4s formed in gene promoters, the structures of their drug complexes have been more difficult to obtain. In 2011 Dai et al. reported the in solution structure of a novel 2:1 complex between a quindoline, a derivative of the natural product cryptolepine, and a major G4 found in the $c$-myc promoter, using the sequence Pu22, in which G14 and G23 are mutated to thymines to isolate the single major conformation [80]. Thus, starting from these structural evidences and following the computational approach of our previous experiences [81,82], we performed a high throughput in silico screening of commercially available alkaloid compounds databases by means of a structure-based approach through docking experiments followed by molecular dynamics simulations against the human telomeric sequence $d\left[\mathrm{AG}_{3}\left(\mathrm{~T}_{2} \mathrm{AG}_{3}\right)_{3}\right]$ and the $c$-myc promoter structure. We identified 69 best hits associated to an improved theoretical binding affinity with respect to that of the active set. Among them, a berberine derivative, already known to remarkably inhibit the telomerase activity, was related to a better theoretical affinity versus c-myc.

\section{Results and Discussion}

\subsection{Structure-Based Virtual Screening of a Database Containing Natural Alkaloids and} Berberine Analogues

In order to identify new natural DNA G4 binders, a structure-based virtual screening of a database containing 26,191 compounds and the application of a series of filters led us to finally identify 21 best hits that showed an improved theoretical binding affinity with respect to a reference berberine derivative (Figure 1).

The database used, containing 26,191 molecules, was built combining libraries of alkaloids and berberine analogues. Subsequently, the filter for drug-like properties, the Lipinski's rule of five and the deduplication led us to globally consider 14,175 compounds. The human telomeric (h-telo) and c-myc promoter oncogene DNA G4s, respectively with PDB codes 3R6R [75] and 2L7V [80], were used to perform the structure-based virtual screening procedure. In particular, Glide Standard Precision (SP) docking simulations [83] were carried out to evaluate the recognition of the 14,175 compounds against DNA G4s. For evaluating the accuracy and reliability of our docking procedure, we firstly performed Glide SP redocking calculations of berberine and quindoline against 3R6R (h-telo) and 2L7V (c-myc) receptors, respectively, in order to geometrically reproduce the experimental data. The obtained poses with the related RMSd values are shown in Figure S1 of the Supplementary Materials.

The best Glide pose for h-telo and $c$-myc DNA G4 receptors was considered and subjected to further analysis. Specifically, the Molecular Mechanics/Generalized Born Surface Area (MM-GBSA) binding free energy [84] was computed. In order to set an energy cut-off applied to choose the best scored ligands, the same docking protocol and binding free energy analysis was carried out for the 4 active compounds quarfloxine, triazine derivative 12459, quindoline and berberine [85-88] (Table 1), chosen on the basis of their known activity towards the target. 


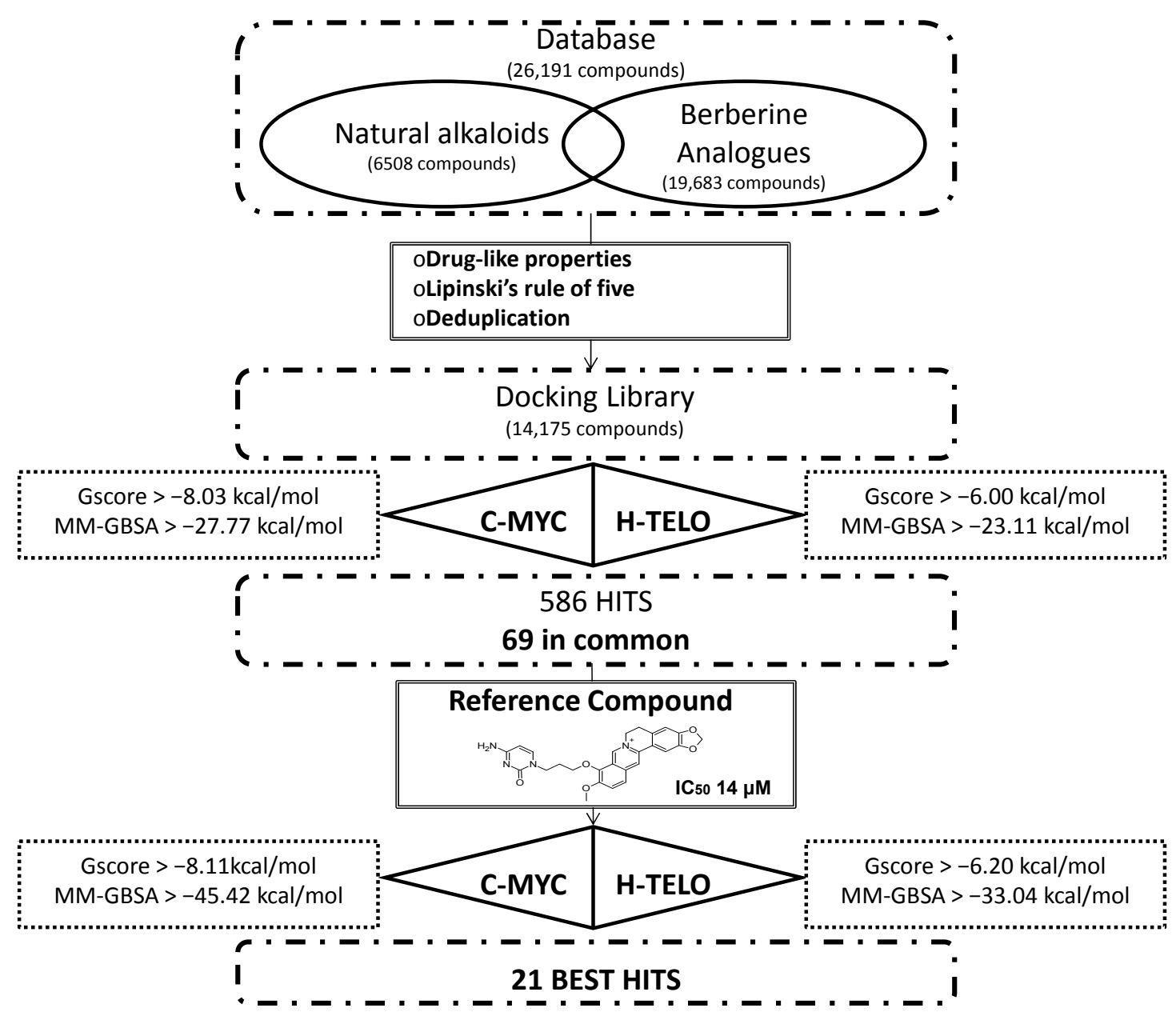

Figure 1. Summary of the theoretical flow chart adopted for the identification of 21 best hits starting from a natural database.

Based on the active compounds free energy of binding, we decided to choose the lowest value of both scoring function on both receptors. Specifically, based on the Glide score (G-score), 1701 compounds with a value lower than $-6.00 \mathrm{kcal} / \mathrm{mol}$ for $h$-telo receptor and 378 ligands with a value lower than $-8.03 \mathrm{kcal} / \mathrm{mol}$ for the $c-m y c$ were selected. All these compounds were filtered on the basis of their MM/GBSA free energy of binding. Within the cut-off value lower than $-23.11 \mathrm{kcal} / \mathrm{mol}$ for $h$-telo and lower than $-27.77 \mathrm{kcal} / \mathrm{mol}$ for $c-m y c, 385$ and 201 compounds were selected, respectively. In order to further filter them, starting from the obtained 586 compounds, we considered only the 69 ligands with a good binding affinity on both h-telo and $c-m y c$ G4s, as reported in Table S1 of the Supplementary Materials. Among these compounds, 54 belong to the berberine derivative class, while the remaining 15 are alkaloids.

Among the 69 best hits, several were associated to antitumor activity, some were Rac GTPases inhibitors and others reported no documented activity (Table S1 of the Supplementary Materials). Interestingly, our virtual screening approach identified two ligands (CID IDs 71165335 and 71476670) found to behave as telomerase inhibitors and actually patent pending. Therefore, even if such a result could represent a validation of our theoretical method, we preferred to discard them from our hits selection, that has been decreased to 67 molecules. 
Table 1. The best Glide score (G-score) and MM-GBSA values obtained after docking simulations of active compounds against $h$-telo (PDB 3R6R) and $c$-myc (PDB 2L7V) G4 receptors. The energy G-scores and MM-GBSA energy values are expressed in $\mathrm{kcal} / \mathrm{mol}$.

\begin{tabular}{|c|c|c|c|c|c|}
\hline \multirow{2}{*}{ Ligands } & \multirow{2}{*}{ Chemical Structure } & \multicolumn{2}{|c|}{ h-telo } & \multicolumn{2}{|c|}{$c-m y c$} \\
\hline & & G-Score & MM-GBSA & G-Score & MM-GBSA \\
\hline Quarfloxine & & -7.67 & -32.28 & -8.98 & -27.77 \\
\hline $\begin{array}{c}\text { Triazine } \\
\text { derivative } 12459\end{array}$ & & -7.49 & -34.17 & -8.90 & -56.60 \\
\hline Quindoline & & -7.00 & -23.11 & -9.35 & -43.00 \\
\hline Berberine & & -6.00 & -26.42 & -8.03 & -33.93 \\
\hline
\end{tabular}

However, since among all the selected compounds only one was found with a documented activity to work as binder of the human telomeric G4 DNA sequence [89], we focused our attention on this ligand (CID ID 44583341), that is reported in Figure 2.<smiles>COc1ccc2cc3[n+](cc2c1OCCCn1ccc(N)nc1=O)CCc1cc2c(cc1-3)OCO2</smiles>

Figure 2. The 2D chemical representation of the reference berberine derivative.

In particular, this $9-O$-substituted berberine derivative, containing an aza-aromatic terminal group, was intensively evaluated by $\mathrm{CD}$ and fluorescence spectroscopy, molecular modeling, FRET-melting assay, PCR stop assay and TRAP assay. The results from biophysical and biochemical assay indicated that the compound possessed much stronger stabilizing effect on the telomeric G4 with respect to berberine [89]. 
The current growing interest in the G4 DNA structures of promoter regions [90,91], in particular versus c-myc, led us to analyze the docking poses obtained on $h$-telo and $c$-myc receptors. With the aim to highlight the differences in the recognition of the used DNA G4 folds, we performed a contact analysis evaluating the interactions in terms of hydrogen bonds and Van der Waal's contacts by means of Maestro graphical interface (Maestro Graphics User Interface, ver. 9.7, Schrödinger LLC, New York, NY, USA, 2014) [92,93] (Table 2). The docking best pose of the reference berberine derivative against the $h$-telo receptor showed its planar surface involved in several end stacking interactions with the G4, G15, G16, G20, G22, T17 and A19 nucleobases. However, due to the presence of a side chain, the ligand was able to establish two hydrogen bonds with the guanine at position 15 and the adenine at position 19 (Figure 3A). Interestingly, in the $c-m y c$ G4 recognition, the compound presented an improved binding affinity and a better interaction pattern compared to h-telo. In particular, the berberine derivative was well stabilized by four hydrogen bonds established with the bases G2 and A3, as shown in Figure 3B. Moreover, the compound was also involved in several Van der Waal's contacts (more than twofold with respect to the h-telo G4), especially with the guanines at position 13 and 8 (Table 2). Using the berberine derivative as reference compound, its G-score and MM-GBSA values were adopted as additional filters of the 69 best hits. In particular, 52 compounds, with h-telo G-score values lower than $-6.20 \mathrm{kcal} / \mathrm{mol}$, and 57 ligands, with $c-m y c$ G-score values lower than $-8.11 \mathrm{kcal} / \mathrm{mol}$, for the $c-m y c$, were selected. Considering the MM-GBSA binding free energies, we decided on 17 and 8 compounds, respectively, for h-telo $(<-33.04 \mathrm{kcal} / \mathrm{mol})$ and $c$-myc $(<-45.42 \mathrm{kcal} / \mathrm{mol}), 4$ of which are common (Table S1 in the Supplementary Materials).
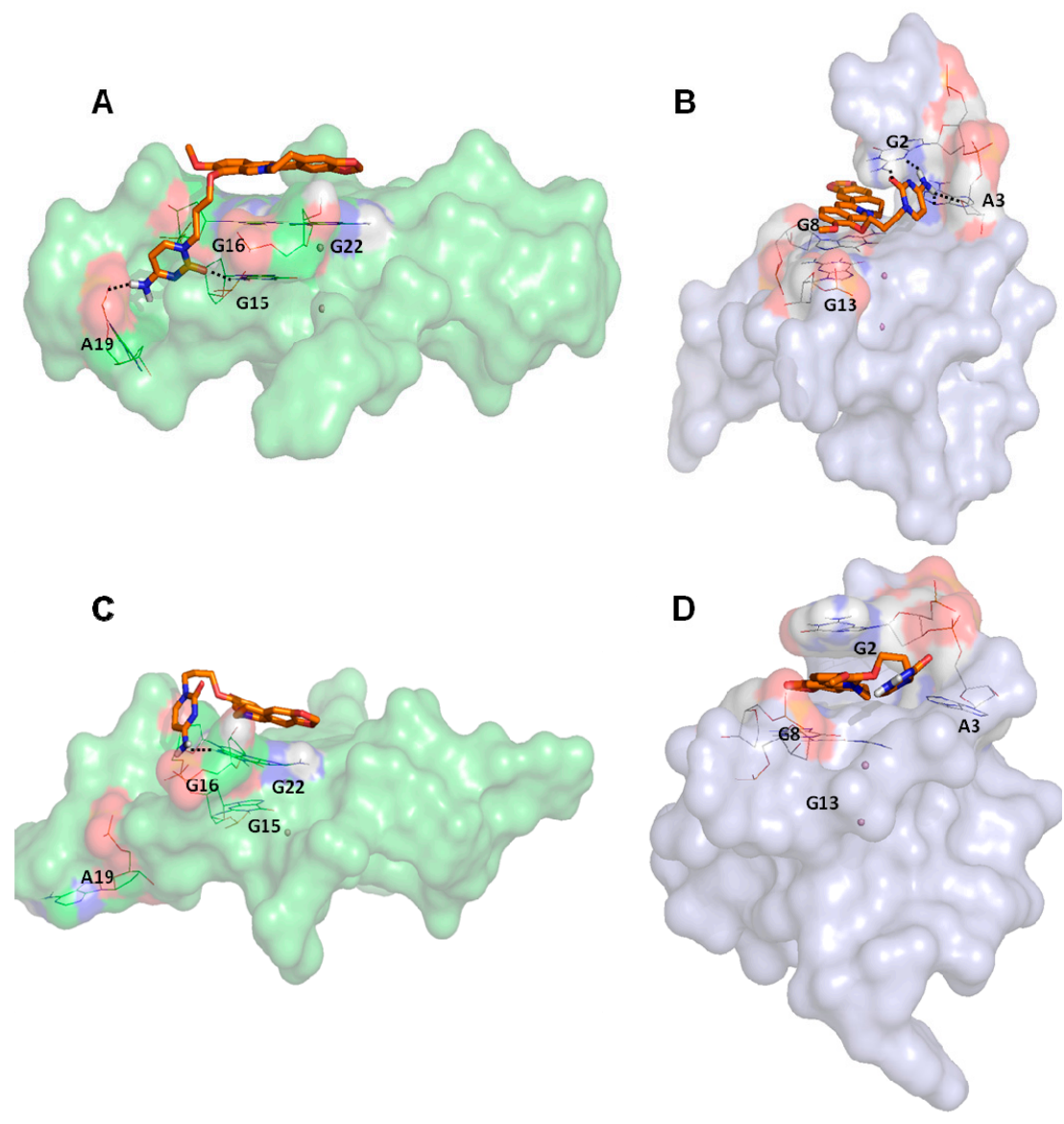

Figure 3. Cont. 

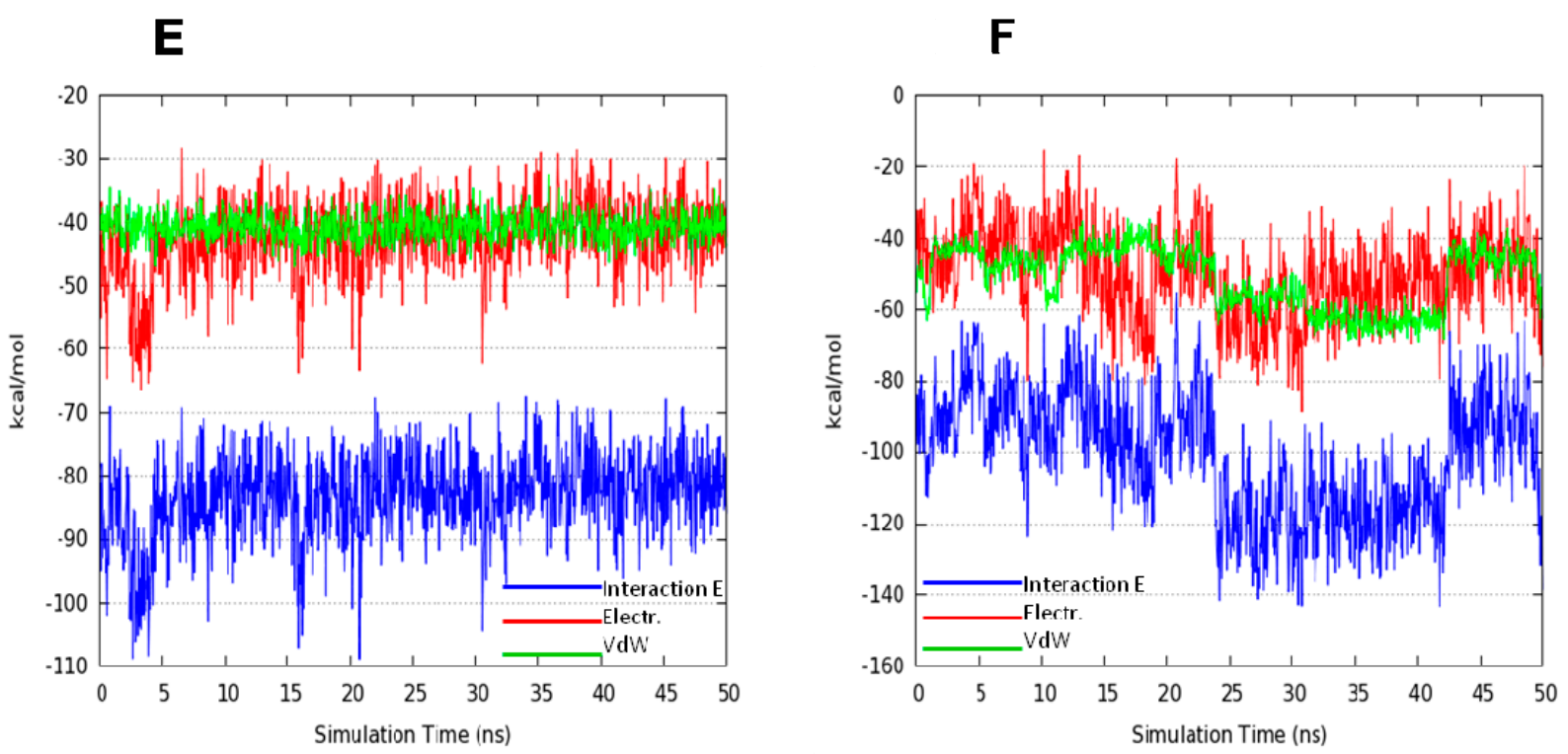

Figure 3. Representation of the best pose after the docking simulations of the berberine derivative against (A) h-telo and (B) c-myc G4 structures. Representation of the most populated structure after the molecular dynamics simulations of the complexes between the berberine derivative and (C) h-telo and (D) c-myc G4 structures. The ligand is shown in orange carbon sticks, the nucleobases involved in hydrogen bonds, reported as black dashed lines, are represented in wireframe, while the coordinating cations are shown as purple spheres. Monitoring of the energy profile after $50 \mathrm{~ns}$ of MDs in terms of Interaction Energy (blue), electrostatic (red) and Van der Waal's (green) contributions, in (E) h-telo and (F) c-myc G4 complexes. The energy values are expressed in $\mathrm{kcal} / \mathrm{mol}$.

Table 2. Global number of contacts and free binding energy values, expressed in $\mathrm{kcal} / \mathrm{mol}$, of the reference berberine derivative complexed to 3R6R (h-telo) and 2L7V (c-myc) models, obtained with the Glide SP approach. HB and GC indicate, respectively, the hydrogen bonds and the good contacts established by the ligand.

\begin{tabular}{|c|c|c|c|c|c|c|c|}
\hline \multicolumn{4}{|c|}{ h-telo } & \multicolumn{4}{|c|}{ c-myc } \\
\hline HB & GC & G-Score & MM-GBSA & HB & GC & G-Score & MM-GBSA \\
\hline 2 & 148 & -6.20 & -33.04 & 4 & 331 & -8.11 & -45.42 \\
\hline
\end{tabular}

In order to further refine the docking results of our reference compound, the best poses on $h$-telo and $c-m y c$ receptors were subjected to molecular dynamics simulations.

\subsection{Molecular Dynamics Simulation (MDs)}

With the aim to explain the binding stability of our reference compound and to clarify the role of its side chain, molecular dynamics simulations (MDs) were performed using NAMD program [94] with the parameters reported in the experimental section. After $50 \mathrm{~ns}$ of MDs of $h$-telo and $c$-myc complexes, the ligand was found to stabilize the G4 core without unfolded structure. Specifically, the Root Mean Square deviation (RMSd) calculated on the guanines core showed the slightly lower average value (equal to $1.20 \AA$ ) for the $c$-myc complex, with respect to that of $h$-telo (RMSd equal to $1.37 \AA$ ). 
Moreover, to better characterize the interactions between the ligand and both G4 receptors, we evaluated the non-bonded interaction energies in terms of electrostatic and Van der Waal's single contributions, using the NAMD Energy Plugin [94]. We observed that, according to the theoretical docking data, the average Interaction Energy value, calculated after $50 \mathrm{~ns}$ of MDs, was equal to $-82.70 \mathrm{kcal} / \mathrm{mol}$ and $-100.54 \mathrm{kcal} / \mathrm{mol}$ for $h$-telo and $c$-myc, respectively.

In particular, it was evident that in c-myc complex, after approximately $25 \mathrm{~ns}$, the Van der Waal's term was associated to the major contribution, accordingly to a sandwiched binding mode between the berberine core and the guanines nucleobases at positions 2, 3, 8 and 13, respectively. Conversely, for the h-telo receptor, the electrostatic and Van der Waal's energetic contributions were almost equivalent (Figure $3 \mathrm{E}, \mathrm{F}$ ) and the complex stability seemed to be related to the formation of only one hydrogen bond between the aza-aromatic side chain of the ligand and the G16 nucleobase, already involved in a stacking contact.

\section{Experimental Section}

\subsection{Database Preparation and Filtering Procedure}

Both natural alkaloids (6508) and berberine analogues (similarity equal to $80 \%, 19,683$ ) libraries were downloaded from PubChem [95] and merged, thus obtaining overall a database of 26,191 compounds. All the molecules included in such database were prepared considering the ionization state at physiological $7.4 \mathrm{pH}$ and then energy minimized using the MMFFs force field as implemented in the LigPrep platform ver. 2.9 [96] of Maestro. Finally, they were filtered basing on their drug-like properties as it has been addressed by the Lipinski's rule of 5 [97] and the duplicated structures were removed. A total amount of 14,175 compounds successfully passed these filters and were subjected to the further molecular recognition process.

\subsection{Receptors Preparation}

The $3 \mathrm{D}$ starting structures of the h-telo $\left[\mathrm{AG}_{3} \mathrm{~T}_{2} \mathrm{AG}_{3} \mathrm{~T}_{2} \mathrm{AG}_{3} \mathrm{~T}_{2} \mathrm{AG}_{3}\right]$ and $c$-myc promoter oncogene [TGAG3 $\mathrm{TG}_{3} \mathrm{TAG}_{3} \mathrm{TG}_{3} \mathrm{TA}_{2}$ ] DNA G4 sequences were downloaded from the Protein Data Bank website [98] with the PDB codes 3R6R [75] and 2L7V [80], respectively. After removing the ligands, both receptors were refined and optimized using the Protein Preparation Wizard tool [99]. Hydrogen atoms were added and the geometry of all the hetero groups was corrected.

\subsection{Receptor Grid Generation and Docking Simulations}

Glide grids were generated by Receptor Grid Generation as implemented in Glide ver. 6.2 [83]. Grid box of both receptors was centered considering the $\mathrm{x}, \mathrm{y}, \mathrm{z}$ coordinates of the G-tetrads centroid and set up wide enough to include the entire macromolecules. The binding affinity for each of the 14175 compounds against h-telo and c-myc G4 sequences was predicted using Glide Standard Precision (SP) protocol, generating ten poses for each ligand. Moreover, the binding free energy was computed by Molecular Mechanics/Generalized Born Surface Area (MM-GBSA) method, VSGB 2.0 continuum dielectric model as solvent model [84], as implemented in Prime [100]. The same docking protocol was adopted for a set of 4 active ligands [85-88]. 


\subsection{Simulation Protocol}

In order to further clarify the binding stability of the reference compound (CID: 44583341) and elucidate the role of its side chain, standard MD simulations were performed with NAMD code ver. 2.9 [94] on both h-telo and c-myc sequences. The parm99 Amber force field, including the recent nucleic acids parmbsc0 parameters [101], was used. Complexes were then placed in a $12.0 \AA$ layer cubic water box using the TIP3P explicit water model. $\mathrm{K}^{+}$cations were added to neutralize the net charge. The SHAKE algorithm was applied to constraint bonds involving hydrogen atoms. A 2 fs integration time step was considered. Ligand charges were computed using the restrained electrostatic potential (RESP) fitting procedure [102]. The ESP was first calculated by means of Jaguar application ver. 8.3 [103] using a $6-31 \mathrm{G}^{*}$ basis set at the Hartree-Fock level of theory. Finally, the RESP charges were calculated by means of the Antechamber module [104].

The system was thus subjected to a double minimization step using the conjugate gradient algorithm in the following conditions: (i) minimization of water molecules and ions, keeping all the solute fixed (2000 steps); (ii) minimization of the entire system, without any restriction (2000 steps). The system was thus equilibrated at $300 \mathrm{~K}$ through $1 \mathrm{~ns}$ in NVT and 1 atm through $1 \mathrm{~ns}$ in NPT ensembles before performing the MD production run of $50 \mathrm{~ns}$ in the NPT ensemble.

\section{Conclusions}

In this work we carried out a high throughput in silico screening of commercially available alkaloids databases by means of a structure-based approach based on docking experiments and molecular dynamics simulations against h-telo and c-myc G4 structures. We identified 69 best hits associated to an improved theoretical binding affinity with respect to that of the active set. Among them, we found a berberine derivative, already known to remarkably inhibit the telomerase activity, related to a better theoretical affinity versus c-myc. This compound, considered as reference ligand, was used to apply a further filter to identify a series of new hits from the used natural database. In particular, the 21 best hits that showed an improved theoretical binding affinity with respect to this reference compound, are currently under experimental investigations in order to complete their biophysical and biological profiles. Moreover, our computational protocol could be applied to other libraries of molecules without a known activity on h-telo and c-myc DNA G4s in order to identify the most promising ones that will be optimized against this emergent target.

\section{Supplementary Materials}

Supplementary materials can be accessed at: http://www.mdpi.com/1420-3049/20/01/0206/s1.

\section{Acknowledgments}

This research is supported by the Italian Ministry of Education (Funding for Investments of Base Research) for the years 2009-2014 (code FIRB-IDEAS RBID082ATK_002), the PRIN 2009 (code 2009MFRKZ8_002). This publication is co-financed by "Commissione Europea, Fondo Sociale Europeo e della Regione Calabria”. R. R. was supported by a POR Calabria FSE 1007-2013 "HEMMAS" 
fellowship. G. C. is grateful for a grant sponsored by the Interregional Research Center for Food Safety and Health at the Magna Græcia University of Catanzaro (MIUR PON a3_00359).

\section{Author Contributions}

RR performed the molecular dynamics simulations; FM and GC carried out the docking experiments; SD was involved in the database preparation and filtering procedure; FO prepared and optimized the used receptors; AA and LP contributed to the draft of the manuscript; SA and EM supervised the manuscript. All authors read and approved the final manuscript.

\section{Conflicts of Interest}

The authors declare no conflict of interest.

\section{References}

1. Chang, C.C.; Kuo, I.C.; Ling, I.F.; Chen, C.T.; Chen, H.C.; Lou, P.J.; Lin, J.J.; Chang, T.C. Detection of quadruplex DNA structures in human telomeres by a fluorescent carbazole derivative. Anal. Chem. 2004, 76, 4490-4494.

2. Palumbo, S.L.; Memmott, R.M.; Uribe, D.J.; Krotova-Khan, Y.; Hurley, L.H.; Ebbinghaus, S.W. A novel G-quadruplex-forming GGA repeat region in the $c-m y b$ promoter is a critical regulator of promoter activity. Nucleic Acids Res. 2008, 36, 1755-1769.

3. Ambrus, A.; Chen, D.; Dai, J.; Jones, R.A.; Yang, D. Solution structure of the biologically relevant G-quadruplex element in the human $c-m y c$ promoter. Implications for G-quadruplex stabilization. Biochemistry 2005, 44, 2048-2058.

4. Phan, A.T.; Kuryavyi, V.; Burge, S.; Neidle, S.; Patel, D.J. Structure of an unprecedented G-quadruplex scaffold in the human c-kit promoter. J. Am. Chem. Soc. 2007, 129, 4386-4392.

5. Chen, Y.; Agrawa, P.; Brown, R.V.; Hatzakis, E.; Hurley, L.; Yang, D. The major G-quadruplex formed in the human platelet-derived growth factor receptor $\beta$ promoter adopts a novel broken-strand structure in $\mathrm{K}^{+}$solution. J. Am. Chem. Soc. 2012, 134, 13220-13223.

6. Dai, J.; Dexheimer, T.S.; Chen, D.; Carver, M.; Ambrus, A.; Jones, R.A.; Yang, D. An intramolecular G-quadruplex structure with mixed parallel/antiparallel G-strands formed in the human BCL-2 promoter region in solution. J. Am. Chem. Soc. 2006, 128, 1096-1098.

7. Kuryavyi, V.; Patel, D.J. Solution structure of a unique G-quadruplex scaffold adopted by a guanosine-rich human intronic sequence. Structure 2010, 18, 73-82.

8. Todd, A.K. Bioinformatics approaches to quadruplex sequence location. Methods 2007, 43, 246-251.

9. Huppert, J.L.; Balasubramanian, S. G-quadruplexes in promoters throughout the human genome. Nucleic Acids Res. 2007, 35, 406-413.

10. Huppert, J.L. Hunting G-quadruplexes. Biochimie 2008, 90, 1140-1148.

11. Todd, A.K.; Johnston, M.; Neidle, S. Highly prevalent putative quadruplex sequence motifs in human DNA. Nucleic Acids Res. 2005, 33, 2901-2907. 
12. Patel, D.J.; Phan, A.T.; Kuryavyi, V. Human telomere, oncogenic promoter and 5'-UTR G-quadruplexes: Diverse higher order DNA and RNA targets for cancer therapeutics. Nucleic Acids Res. 2007, 35, 7429-7455.

13. Eddy, J.; Maizels, N. Gene function correlates with potential for G4 DNA formation in the human genome. Nucleic Acids Res. 2006, 34, 3887-3896.

14. Biffi, G.; Tannahill, D.; McCafferty, J.; Balasubramanian, S. Quantitative visualization of DNA G-quadruplex structures in human cells. Nat. Chem. 2013, 5, 182-186.

15. Cui, X.; Yuan, G. Formation and recognition of G-quadruplex in promoter of c-myb oncogene by electrospray ionization mass spectrometry. J. Mass Spectrom. 2011, 46, 849-855.

16. Lin, S.; Li, S.; Chen, Z.; He, X.; Zhang, Y.; Xu, X.; Xu, M.; Yuan, G. Formation, recognition and bioactivities of a novel G-quadruplex in the STAT3 gene. Bioorg. Med. Chem. Lett. 2011, 21, 5987-5991.

17. Tan, W.; Yuan, G. Electrospray ionization mass spectrometric exploration of the high-affinity binding of three natural alkaloids with the mRNA G-quadruplex in the BCL2 5'-untranslated region. Rapid Commun. Mass Spectrom. 2013, 27, 560-564.

18. Alcaro, S. The impact of the G-quadruplex conformation in the development of novel therapeutic and diagnostic agents. Curr. Pharm. Des. 2012, 18, 1867-1872.

19. Burge, S.; Parkinson, G.N.; Hazel, P.; Todd, A.K.; Neidle, S. Quadruplex DNA: Sequence, topology and structure. Nucleic Acids Res. 2006, 34, 5402-5415.

20. Ou, T.M.; Lu, Y.J.; Tan, J.H.; Huang, Z.S.; Wong, K.Y.; Gu, L.Q. G-Quadruplexes: Targets in Anticancer Drug Design. ChemMedChem 2008, 3, 690-713.

21. Parrotta, L.; Ortuso, F.; Moraca, F.; Rocca, R.; Costa, G.; Alcaro, S.; Artese, A. Targeting unimolecular G-quadruplex nucleic acids: A new paradigm for the drug discovery? Expert Opin. Drug Discov. 2014, 9, 1167-1187.

22. Sattin, G.; Artese, A.; Nadai, M.; Costa, G.; Parrotta, L.; Alcaro, S.; Palumbo, M.; Richter, S.N. Conformation and stability of intramolecular telomeric g-quadruplexes: Sequence effects in the loops. PLoS One 2013, 8, e84113.

23. Sundquist, W.; Klug, A. Telomeric DNA dimerizes by formation of guanine tetrads between hairpin loops. Nature 1989, 342, 825-829.

24. Simonsson, T. G-quadruplex DNA structures-Variations on a theme. Biol. Chem. 2001, 382, 621-628.

25. Zahler, A.M.; Williamson, J.R.; Cech, T.R.; Prescott, D.M. Inhibition of telomerase by G-quartet DNA structures. Nature 1991, 350, 718-720.

26. Franceschin, M. G-quadruplex DNA structures and organic chemistry: More than one connection. Eur. J. Org. Chem. 2009, 14, 2225-2238.

27. Hurley, L.H. DNA and its associated processes as targets for cancer therapy. Nat. Rev. Cancer 2002, 2, 188-200.

28. Neidle, S.; Parkinson, G. Telomere maintenance as a target for anticancer drug discovery. Nat. Rev. Drug Discov. 2002, 1, 383-393.

29. Riou, J.F. G-quadruplex interacting agents targeting the telomeric G-overhang are more than simple telomerase inhibitors. Curr. Med. Chem. Anticancer Agents 2004, 4, 439-443. 
30. Bearss, D.J.; Hurley, L.H.; Von Hoff, D.D. Telomere maintenance mechanisms as a target for drug development. Oncogene 2000, 19, 6632-6641.

31. Alcaro, S.; Artese, A.; Iley, J.N.; Maccari, R.; Missailidis, S.; Ortuso, F.; Ottana, R.; Ragazzon, P.; Vigorita, M.G. Tetraplex DNA specific ligands based on the fluorenone-carboxamide scaffold. Bioorg. Med. Chem. Lett. 2007, 17, 2509-2514.

32. Alcaro, S.; Artese, A.; Iley, J.N.; Missailidis, S.; Ortuso, F.; Parrotta, L.; Pasceri, R.; Paduano, F.; Sissi, C.; Trapasso, F.; et al. Rational design, synthesis, biophysical and antiproliferative evaluation of fluorenone derivatives with DNA G-quadruplex binding properties. ChemMedChem 2010, 5, $575-583$.

33. Alcaro, S.; Artese, A.; Costa, G.; Distinto, S.; Ortuso, F.; Parrotta, L. Conformational studies and solvent-accessible surface area analysis of known selective DNA G-quadruplex. binders. Biochimie 2011, 93, 1267-1274.

34. Doria, F.; Nadai, M.; Folini, M.; Di Antonio, M.; Germani, L.; Percivalle, C.; Sissi, C.; Zaffaroni, N.; Alcaro, S.; Artese, A.; et al. Hybrid ligand-alkylating agents targeting telomeric G-quadruplex structures. Org. Biomol. Chem. 2012, 10, 2798-2806.

35. Milelli, A.; Tumiatti, V.; Micco, M.; Rosini, M.; Zuccari, G.; Raffaghello, L.; Bianchi, G.; Pistoia, V.; Fernando Díaz, J.; Pera, B.; et al. Structure-activity relationships of novel substituted naphthalene diimides as anticancer agents. Eur. J. Med. Chem. 2012, 57, 417-428.

36. Artese, A.; Parrotta, L.; Alcaro, S.; Ortuso, F.; Costa, G.; Sissi, C. Molecular recognition of human telomeric DNA by phenanthroline-based G-quadruplex ligands. Open J. Med. Chem. 2013, 3, 41-49.

37. Artese, A.; Costa, G.; Distinto, S.; Moraca, F.; Ortuso, F.; Parrotta, L.; Alcaro, S. Toward the design of new DNA G-quadruplex ligands through rational analysis of polymorphism and binding data. Eur. J. Med. Chem. 2013, 68, 139-149.

38. Percivalle, C.; Sissi, C.; Greco, M.L.; Musetti, C.; Mariani, A.; Artese, A.; Costa, G.; Perrore, M.L.; Alcaro, S.; Freccero, M. Aryl ethynyl anthraquinones: A useful platform for targeting telomeric G-quadruplex structures. Org. Biomol. Chem. 2014, 12, 3744-3754.

39. Ruden, M.; Puri, N. Novel anticancer therapeutics targeting telomerase. Cancer Treat. Rev. 2013, 39, 444-456.

40. Alcaro, S.; Costa, G.; Distinto, S.; Moraca, F.; Ortuso, F.; Parrotta, L.; Artese, A. The Polymorfisms of DNA G-Quadruplex investigated by Docking Experiments with Telomestatin Enantiomers. Curr. Pharm. Des. 2012, 18, 1867-1872.

41. Qin, Y.; Hurley, L.H. Structures, folding patterns, and functions of intramolecular DNA G-quadruplexes found in eukaryotic promoter regions. Biochimie 2008, 90, 1149-1171.

42. Dang, C.V.; Resar, L.M.; Emison, E.; Kim, S.; Li, Q.; Prescott, J.E.; Wonsey, D.; Zeller, K. Function of the c-Myc oncogenic transcription factor. Exp. Cell Res. 1999, 253, 63-77.

43. Gonzalez, V.; Hurley, L.H. The c-MYC NHE III(1): Function and regulation. Annu. Rev. Pharmacol. Toxicol. 2010, 50, 111-129.

44. Tomonaga, T.; Levens, D. Activating transcription from single stranded DNA. Proc. Natl. Acad. Sci. USA 1996, 93, 5830-5835.

45. Simonsson, T.; Pecinka, P.; Kubista, M. DNA tetraplex formation in the control region of $c-m y c$. Nucleic Acids Res. 1998, 26, 1167-1172. 
46. Siddiqui-Jain, A.; Grand, C.L.; Bearss, J.; Hurley, L.H. Direct evidence for a G-quadruplex in a promoter region and its targeting with a small molecule to repress c-MYC transcription. Proc. Natl. Acad. Sci. USA 2002, 99, 11593-11598.

47. Brooks, T.A.; Hurley, L.H. The role of supercoiling in transcriptional control of MYC and its importance in molecular therapeutics. Nat. Rev. Cancer 2009, 9, 849-861.

48. Yang, D.; Hurley, L.H. Structure of the biologically relevant G-quadruplex in the $c-m y c$ promoter. Nucleosides Nucleotides Nucleic Acids 2006, 25, 951-968.

49. Grand, C.L.; Han, H.; Munoz, R.M.; Weitman, S.; Von Hoff, D.D.; Hurley, L.H.; Bearss, D.J. The cationic porphyrin TMPyP4 down-regulates c-MYC and human telomerase reverse transcriptase expression and inhibits tumor growth in vivo. Mol. Cancer Ther. 2002, 1, 565-673.

50. Tan, W.; Zhou, J.; Yuan, G. Electrospray ionization mass spectrometry probing of binding affinity of berbamine, a flexible cyclic alkaloid from traditional Chinese medicine, with G-quadruplex DNA. Rapid Commun. Mass Spectrom. 2014, 28, 143-147.

51. Lee, H.M.; Chan, D.S.; Yang, F.; Lam, H.Y.; Yan, S.C.; Che, C.M.; Ma, D.L.; Leung, C.H. Identification of natural product Fonsecin B as a stabilizing ligand of $c-m y c$ G-quadruplex DNA by high-throughput virtual screening. Chem. Commun. 2010, 46, 4680-4682.

52. Chan, D.S; Lee, H.M.; Yang, F.; Che, C.M.; Wong, C.C.; Abagyan, R.; Leung, C.H.; Ma, D.L. Structure-based discovery of natural-product-like TNF-alpha inhibitors. Angew. Chem. Int. Ed. 2010, 49, 2860-2864.

53. Leung, C.H.; Chan, D.S.; Yang, H.; Abagyan, R.; Lee, S.M.; Zhu, G.Y.; Fong, W.F.; Ma, D.L. A natural product-like inhibitor of NEDD8-activating enzyme. Chem. Commun. 2011, 47, 2511-2513.

54. Zhong, H.J.; Ma, V.P.; Cheng, Z.; Chan, D.S.; He, H.Z.; Leung, K.H.; Ma, D.L.; Leung, C.H. Discovery of a natural product inhibitor targeting protein neddylation by structure-based virtual screening. Biochimie 2012, 94, 2457-2460.

55. Ma, D.L.; Chan, D.S.; Leung, C.H. Drug repositioning by structure-based virtual screening. Chem. Soc. Rev. 2013, 42, 2130-2141.

56. Liu, L.J.; Leung, K.H.; Chan, D.S.; Wang, Y.T.; Ma, D.L.; Leung, C.H. Identification of a natural product-like STAT3 dimerization inhibitor by structure-based virtual screening. Cell Death Dis. 2014, 5, e1293.

57. Ma, D.L.; Chan, D.S.; Fu, W.C.; He, H.Z.; Yang, H.; Yan, S.C.; Leung, C.H. Discovery of a natural product-like c-myc G-quadruplex DNA groove-binder by molecular docking. PLoS One 2012, 7, e43278.

58. Newman, D.J.; Cragg, G.M. Natural products as sources of new drugs over the 30 years from 1981 to 2010. J. Nat. Prod. 2012, 75, 311-335.

59. Selvi, B.R.; Pradhan, S.K.; Shandilya, J.; Das, C.; Sailaja, B.S.; Shankar, G.N.; Gadad, S.S.; Reddy, A.; Dasgupta, D.; Kundu, T.K. Sanguinarine interacts with chromatin, modulates epigenetic modifications, and transcription in the context of chromatin. Chem. Biol. 2009, 16, 203-216.

60. Ghosh, S.; Pradhan, S.K.; Kar, A.; Chowdhury, S.; Dasgupta, D. Molecular basis of recognition of quadruplexes human telomere and c-myc promoter by the putative anticancer agent sanguinarine. Biochim. Biophys. Acta 2013, 1830, 4189-4201.

61. Ji, X.; Sun, H.; Zhou, H.; Xiang, J.; Tang, Y.; Zhao, C. The interaction of telomeric DNA and C-myc22 G-quadruplex with 11 natural alkaloids. Nucleic Acid Ther. 2012, 22, 127-136. 
62. Qin, Y.; Pang, J.Y.; Chen, W.H.; Zhao, Z.Z.; Liu, L.; Jiang, Z.H. Inhibition of DNA topoisomerase I by natural and synthetic mono- and dimeric protoberberine alkaloids. Chem. Biodivers. 2007, 4, 481-487.

63. Kim, S.A.; Kwon, Y.; Kim, J.H.; Muller, M.T.; Chung, I.K. Induction of topoisomerase II-mediated DNA cleavage by a protoberberine alkaloid, berberrubine. Biochemistry 1998, 37, 16316-16324.

64. Kang, M.R.; Chung, I.K. Down-regulation of DNA topoisomerase IIalpha in human colorectal carcinoma cells resistant to a protoberberine alkaloid, berberrubine. Mol. Pharmacol. 2002, 61, 879-884.

65. Bessi, I.; Bazzicalupi, C.; Richter, C.; Jonker, H.R.; Saxena, K.; Sissi, C.; Chioccioli, M.; Bianco, S.; Bilia, A.R.; Schwalbe, H.; et al. Spectroscopic, molecular modeling, and NMR-spectroscopic investigation of the binding mode of the natural alkaloids berberine and sanguinarine to human telomeric G-quadruplex DNA. ACS Chem. Biol. 2012, 7, 1109-1119.

66. Gornall, K.C.; Samosorn, S.; Talib, J.; Bremner, J.B.; Beck, J.L. Selectivity of an indolyl berberine derivative for tetrameric G-quadruplex DNA. Rapid Commun. Mass Spectrom. 2007, 21, 1759-1766.

67. Franceschin, M.; Rossetti, L.; D’Ambrosio, A.; Schirripa, S.; Bianco, A.; Ortaggi, G.; Savino, M.; Schultes, C.; Neidle, S. Natural and synthetic G-quadruplex interactive berberine derivatives. Bioorg. Med. Chem. Lett. 2006, 16, 1707-1711.

68. Zhang, W.J.; Ou, T.M.; Lu, Y.J.; Huang, Y.Y.; Wu, W.B.; Huang, Z.S.; Zhou, J.L.; Wong, K.Y.; $\mathrm{Gu}$, L.Q. 9-Substituted berberine derivatives as G-quadruplex stabilizing ligands in telomeric DNA. Bioorg. Med. Chem. 2007, 15, 5493-5501.

69. Ma, Y.; Ou, T.M.; Hou, J.Q.; Lu, Y.J.; Tan, J.H.; Gu, L.Q.; Huang, Z.S. 9-N-Substituted berberine derivatives: Stabilization of G-quadruplex DNA and down-regulation of oncogene c-myc. Bioorg. Med. Chem. 2008, 16, 7582-7591.

70. Ma, Y.; Ou, T.M.; Tan, J.H.; Hou, J.Q.; Huang, S.L.; Gu, L.Q.; Huang, Z.S. Quinolino-benzo[5,6]-dihydroisoquindolium compounds derived from berberine: A new class of highly selective ligands for G-quadruplex DNA in c-myc oncogene. Eur. J. Med. Chem. 2011, 46, 1906-1913.

71. Bournine, L.; Bensalem, S.; Wauters, J.N.; Iguer-Ouada, M.; Maiza-Benabdesselam, F.; Bedjou, F.; Castronovo, V.; Bellahcène, A.; Tits, M.; Frédérich, M. Identification and quantification of the main active anticancer alkaloids from the root of Glaucium flavum. Int. J. Mol. Sci. 2013, 14, 23533-23544.

72. Cui, X.; Lin, S.; Zhou, J.; Yuan, G. Investigation of non-covalent interaction of natural flexible cyclic molecules with telomeric RNA G-quadruplexes by electrospray ionization mass spectrometry. Rapid Commun. Mass Spectrom. 2012, 26, 1803-1809.

73. Aniszewski, T. Alkaloids: Chemical and Biological Perspectives. In Alkaloids-Secrets of Life: Aklaloid Chemistry, Biological Significance, Applications and Ecological Role, 1 ed.; Elsevier Science: Amsterdam, The Netherlands, 2007; p. 334.

74. Bhadra, K.; Kumar, G.S. Interaction of berberine, palmatine, coralyne, and sanguinarine to quadruplex DNA: A comparative spectroscopic and calorimetric study. Biochim. Biophys. Acta 2011, 1810, 485-496. 
75. Bazzicalupi, C.; Ferraroni, M.; Bilia, A.R.; Scheggi, F.; Gratteri, P. The crystal structure of human telomeric DNA complexed with berberine: An interesting case of stacked ligand to G-tetrad ratio higher than 1:1. Nucleic Acids Res. 2013, 41, 632-638.

76. Yang, D.; Okamoto, K. Structural insights into G-quadruplexes: Towards new anticancer drugs. Future Med. Chem. 2010, 2, 619-646.

77. Dai, J.; Chen, D.; Jones, R.A.; Hurley, L.H.; Yang, D. NMR solution structure of the major G-quadruplex structure formed in the human BCL2 promoter region. Nucleic Acids Res. 2006, 34, 5133-5144.

78. Dai, J.; Carver, M.; Punchihewa, C.; Jones, R.A.; Yang, D. Structure of the Hybrid-2 type intramolecular human telomeric G-quadruplex in $\mathrm{K}^{+}$solution: Insights into structure polymorphism of the human telomeric sequence. Nucleic Acids Res. 2007, 35, 4927-4940.

79. De Cian, A.; Lacroix, L.; Douarre, C.; Temime-Smaali, N.; Trentesaux, C.; Riou, J.F.; Mergny, J.L. Targeting telomeres and telomerase. Biochimie 2008, 90, 131-155.

80. Dai, J.; Carver, M.; Hurley, L.H.; Yang, D. Solution structure of a 2:1 quindoline-c-myc G-quadruplex: Insights into G-quadruplex-interactive small molecule drug design. J. Am. Chem. Soc. 2011, 133, 17673-17680.

81. Artese, A.; Costa, G.; Ortuso, F.; Parrotta, L.; Alcaro, S. Identification of new natural DNA G-quadruplex binders selected by a structure-based virtual screening approach. Molecules 2013, 18, 12051-12070.

82. Alcaro, S.; Musetti, C.; Distinto, S.; Casatti, M.; Zagotto, G.; Artese, A.; Parrotta, L.; Moraca, F.; Costa, G.; Ortuso, F.; et al. Identification and characterization of new DNA G-quadruplex binders selected by a combination of ligand and structure based virtual screening approaches. J. Med. Chem. 2013, 56, 843-855.

83. Glide, version 6.2; Schrödinger, LLC: New York, NY, USA, 2014.

84. Afzal, O.; Kumar, S.; Kumar, R.; Firoz, A.; Jaggi, M.; Bawa, S. Docking based virtual screening and molecular dynamics study to identify potential monoacylglycerol lipase inhibitors. Bioorg. Med. Chem. Lett. 2014, 24, 3986-3996.

85. Drygin, D.; Siddiqui-Jain, A.; O’Brien, S.; Schwaebe, M.; Lin, A.; Bliesath, J.; Ho, C.B.; Proffitt C.; Trent, K.; Whitten, J.P.; et al. Anticancer activity of CX-3543: A direct inhibitor of rRNA biogenesis. Cancer Res. 2009, 69, 7653-7561.

86. Gomez, D.; Lemarteleur, T.; Lacroix, L.; Mailliet, P.; Mergny, J.L.; Riou, J.F. Telomerase downregulation induced by the G-quadruplex ligand 12459 in A549 cells is mediated by TERT RNA alternative splicing. Nucleic Acids Res. 2004, 32, 371-379.

87. Ou, T.M.; Lu, Y.J.; Zhang, C.; Huang, Z.S.; Wang, X.D.; Tan, J.H.; Chen, Y.; Ma, D.L.; Wong, K.Y.; Tang, J.C.; et al. Stabilization of G-quadruplex DNA and down-regulation of oncogene c-myc by quindoline derivatives. J. Med. Chem. 2007, 50, 1465-1474.

88. Naasani, I.; Seimiya, H.; Yamori, T.; Tsuruo, T. FJ5002: A potent telomerase inhibitor identified by exploiting the disease-oriented screening program with compare analysis. Cancer Res. 1999, 59, 4004-4011.

89. Ma, Y.; Ou, T.M.; Tan, J.H.; Hou, J.Q.; Huang, S.L.; Gu, L.Q.; Huang, Z.S. Synthesis and evaluation of 9-O-substituted berberine derivatives containing aza-aromatic terminal group as highly selective telomeric G-quadruplex stabilizing ligands. Bioorg. Med. Chem. Lett. 2009, 19, 3414-3417. 
90. Chen, B.J.; Wu, Y.L.; Tanaka, Y.; Zhang, W. Small Molecules Targeting c-Myc Oncogene: Promising Anti-Cancer Therapeutics. Int. J. Biol. Sci. 2014, 10, 1084-1096.

91. Hassani, L.; Fazeli, Z.; Safaei, E.; Rastegar, H.; Akbari, M. A spectroscopic investigation of the interaction between c-MYC DNA and tetrapyridinoporphyrazinatozinc(II). J. Biol. Phys. 2014, 40, 275-283.

92. Maestro 9.7; Schrödinger, LLC: New York, NY, USA, 2014.

93. Mohamadi, F.; Richards, N.G.J.; Guida, W.C.; Liskamp, R.; Lipton, M.; Caufield, C.; Chang, G.; Hendrickson, T.; Still, W.C. Macromodel-an integrated software system for modeling organic and bioorganic molecules using molecular mechanics. J. Comput. Chem. 1990, 11, 440-467.

94. Phillips, J.C.; Braun, R.; Wang, W.; Gumbart, J.; Tajkhorshid, E.; Villa, E.; Chipot, C.; Skeel, R.D.; Kale, L.; Schulten, K. Scalable molecular dynamics with NAMD. J. Comput. Chem. 2005, 26, 1781-1802.

95. PubChem. Available online: http://pubchem.ncbi.nlm.nih.gov/ (accessed on 1 November 2014).

96. LigPrep, version 2.9; Schrödinger, LLC: New York, NY, USA, 2014.

97. Lipinski, C.A. Lead- and drug-like compounds: The rule-of-five revolution. Drug Discov. Today Technol. 2004, 1, 337-341.

98. The Research Collaboratory for Structural Bioinformatics (RCSB) Protein Data Bank (PDB). Available online: http://www.rcsb.org (accessed on 1 November 2014).

99. Protein Preparation Wizard; Schrödinger, LLC: New York, NY, USA, 2014.

100. Prime, version 3.5; Schrödinger, LLC: New York, NY, USA, 2014.

101. Pérez, A.; Marchàn, I.; Svozil, D.; Sponer, J.; Cheatham, T.E., 3rd; Laughton, C.A.; Orozco, M. Refinement of the AMBER Force Fields for Nucleic Acids: Improving the Description of $\alpha / \gamma$ Conformers. Biophys. J. 2007, 92, 3817-3829.

102. Wang, J.; Cieplak, P.; Kollman, P.A. How Well Does a Restrained Electrostatic Potential (RESP) Model Perform in Calculating Conformational Energies of Organic and Biological Molecules? J. Comput. Chem. 1999, 21, 1049-1074.

103. Jaguar, version 8.3; Schrödinger, LLC: New York, NY, USA, 2014.

104. Wang, J.; Wang, W.; Kollman, P.A.; Case, D.A. Automatic atom type and bond type perception in molecular mechanical calculations. J. Mol. Graph. Model. 2006, 25, 247-260.

Sample Availability: Samples of the compounds are not available from the authors.

(C) 2014 by the authors; licensee MDPI, Basel, Switzerland. This article is an open access article distributed under the terms and conditions of the Creative Commons Attribution license (http://creativecommons.org/licenses/by/4.0/). 